\title{
LA APLICACIÓN \\ DE LA NORMA \\ ÉTICA UNIVERSAL \\ (KARL-OTTO APEL) \\ Mario Ramirez*
}

La exigencia de "aplicación" del principio ético universal constituye y determina la especificidad de la "ética discursiva" de Karl-Otto Apel respecto a toda ética de principios meramente formalista. Trataremos de ubicar los fundamentos de esta tesis así como ciertos problemas límites del tema de la "aplicación".

\section{Universalidad y aplicabilidad}

Podemos decir que la ética discursiva o ética dialógica de Apel consiste en la afirmación simultánea de dos principios a priori: el principio de universalización y el principio de aplicabilidad. La norma ética básica debe ser universalizable y aplicable; fundamentable y realizable. Tan inmoral es la "norma parcial" fácilmente aplicable del relativista, como la "norma universal" inaplicable o aplicable a discreción del idealista. De esa manera, el imperativo categórico de una ética discursiva es doble: nos ordena asumir el principio de universalización y, a la vez, nos ordena buscar las condiciones efectivas de su aplicación. No sólo nos exige que nos comprometemos moralmente, nos exige además que cuando las

\footnotetext{
* Universidad Michoacana.
} 
Mario Ramírez

condiciones no nos permitan hacerlo, nos comprometamos a buscar las condiciones que sí lo permitan. La conjunción de ambas exigencias delimita la naturaleza última de la ética discursiva en cuanto ética de la responsabilidad. Es así como podemos enfrentar la disyunción que nos impele a elegir bien una norma moralmente incuestionable pero impráctica o bien una norma aplicable aunque moralmente irrelevante.

Nuestra tesis insiste, pues, en que las dos partes de la ética, tal y como han sido determinadas por Apel-parte "A" de la fundamentación y parte " $B$ " de la aplicación- configuran una totalidad interdependiente que define el carácter propio y último de la ética discursiva. De esta manera, la pregunta ético-crítica: ¿es posible una ética universal? debe ser desglosada en dos momentos o aspectos: 1) ies posible teóricamente?, lo que nos remite al problema de si es racionalmente fundamentable; y 2) ¿es posible prácticamente?, que nos remite a las condiciones de su aplicación efectiva. El carácter crítico radical de la ética discursiva consiste en la necesidad de considerar simultáneamente ambos momentos de la pregunta. Esto significa que ya el tratamiento del primer momento prefigura la necesidad y posibilidad del segundo; en otras palabras: que la explicación del principio de universalización se realiza, y ha de realizarse, en el horizonte del problema de la aplicación. Tal explicación se presenta, así, bajo la forma de una fundamentación pragmática y comunitaria.

\section{El principio de universalización}

Inicialmente, la fundamentacion pragmática se distingue de la fundamentación formal clásica (kantiana) en su ampliación de la dimensión "sintética" del principio a priori. Podríamos decir que para Apel lo sintético ya no se refiere a la "pluralidad empírica de datos" para una conciencia solipsista (al 'Yo pienso'), sino, en tanto hablamos estrictamente de experiencia moral, a la pluralidad de conciencias para una comunidad intersubjetiva lingüísticamente mediada (al 'Nosotros hablamos'). El principio ético no sólo es formalmente universal, sino también materialmente universal (aunque en un sentido trascendental); no sólo 


\section{LA NORMA ÉTICA UNIVERSAL}

es universal por su objetivo sino, todavía más, por sus condiciones. Ya no puede enunciarse de manera puramente abstracta como "válido para todos" (y cada uno), ha de enunciarse de manera "sintética" como "válido para todos por validado por todos", para la humanidad como "totalidad de interrelación comunitaria posible" y no sólo como conjunto de todos los individuos que analíticamente satisfacen el predicado de "racionalidad".

La comunidad humana -"real" y "posible"-es el sujeto y el objeto, la condición y la consecuencia, del principio ético universal. Mientras que para Kant la exigencia de someter nuestras máximas a la prueba de la universalización (considerar que nuestra máxima es una Ley de la Naturaleza) podía ser realizada reflexiva y analíticamente por el sujeto solipsista, para Apel la prueba de universalización ya no se plantea como un procedimiento lógico-formal e intra-subjetivo sino como la exigencia de someter nuestras máximas a la discusión pública y a la argumentación inter-subjetiva. De esta manera, para Apel como para Habermas, ${ }^{1}$ la norma válida universalmente es aquella en cuya definición participan los sujetos interesados y en la que se consideran las consecuencias de su aplicación para todos los afectados posibles.

Ahora bien, según Apel, el proceso de universalización, en tanto proceso práctico y efectivo aunque ilimitado y abierto, no puede ser reducido a los procesos particulares del diálogo en la comunidad real, pues todo proceso comunicativo concreto es necesariamente parcial y se encuentra sometido a una serie de instancias que obstaculizan, limitan o imposibilitan el seguimiento por los participantes de las reglas de la argumentación adecuada -normas lógicas, disposición a reconocer el mejor argumento, relación de reciprocidad y respeto entre los argumentantes-, condiciones de posibilidad de un acto comunicativo auténtico exento de retórica, manipulación o frivolidad. Es por esto que el diálogo argumentativo y la búsqueda del consenso racional -acuerdo o entendimiento intersubjetivo que se atiene a la fuerza no coactiva del mejor

${ }^{1}$ Cf. Jürgen Habermas, "Etica del discurso. Notas sobre un programa de fundamentación", en Conciencia moral y acción comunicativa, Ed. Península, Barcclona, 1985, p. 117. 
Mario Ramírez

argumento- requiere el postulado de una "comunidad ideal de comunicación". Para Apel, tal postulado no es un mero "ideal" o una "ilusión", es, por el contrario, el presupuesto pragmático-trascendental irrebasable de todo acto comunicativo: la comunicación no sería posible sin él. Posee, por tanto, carácter de obligatoriedad para todo "dialogante". Desde que argumento, desde que dialogo, incluso desde que hablo, presupongo ya la posibilidad de alcanzar un acuerdo racional y, por tanto, la comunidad ideal de comunicación. Según el enfoque pragmático de Apel y Habermas, el enunciado más elemental-actual o virtual-implica necesariamente, en tanto "acto de habla" (ilocución), "pretensiones de validez" que se encuentran satisfechas, o pueden o podrían serlo, mediante el proceso comunicativo y argumentativo.

El presupuesto de la posibilidad del acuerdo racional se convierte así, según Apel, en la norma ética básica para el que argumenta y para todo ser humano en tanto ser racional (en tanto ser capaz de competencia lingüística y competencia comunicativa); es, por tanto, una norma ética universal, válida para todo ser humano en todos los niveles de su com70 portamiento social y no sólo para ciertos aspectos o niveles de la experiencia (el discurso teórico o el diálogo formal y explícito). La comunidad "ideal" es un principio trascendental, una "idea regulativa", que es -pragmáticamente-y ha de ser-reflexiva y éticamente-anticipada contrafácticamente por toda comunidad real de comunicación.

Ahora bien, dado que el hombre se encuentra necesariamente sometido a exigencias prácticas concretas (principio de sobrevivencia, autoafirmación de las instituciones establecidas, intereses estratégicos), la realización de la comunidad ideal sólo puede postularse como un proceso problemático, asintótico y abierto, aunque siempre posible y necesario, siempre irrenunciable. Como hemos dicho, el problema de la realización de la comunidad ideal, el problema de la aplicación de la norma ética básica, se convierte en el problema clave de la reflexión ética. No es un asunto secundario y contingente respecto a la cuestión de la fundamentación; por el contrario: la posibilidad real de la vida ética es la prueba última de la validez del proceso de fundamentación y de la efectividad de su racionalidad. No nos estamos refiriendo a la realización empírica del "comportamiento moral" como prueba de la validez de la norma ética. 


\section{LA NORMA ÉTICA UNIVERSAL}

Ciertamente, de acuerdo con la argumentación apriorística de Apel, ningún "hecho moral" puede probar o refutar la validez de una norma, pues estaríamos confundiendo el ser de la "norma" (que es prescriptiva) con un "concepto" (que es descriptivo o explicativo). Nos referimos más bien a la posibilidad para toda vida actual y toda comunidad real de advenir a la existencia ética. El planteamiento de tal posibilidad es lo que convierte a la ética discursiva en una ética de la responsabilidad, una ética de la "aplicación" y de la "transformación".

\section{La responsabilidad}

En tanto ética universal, la ética discursiva continúa la herencia kantiana, pero modificándola y transformándola. Pasamos de un principio de reciprocidad universal sólo supuesto y asentado por el sujeto monológico, a un principio de reciprocidad comunitariamente construido en el diálogo argumentativo. Esto es, pasamos de una ética subjetivista, de la buena intención o de la convicción, en la que se eliminan por heterónomas todas las consideraciones acerca de las consecuencias y subsecuencias de una decisión, y finalmente, de la misma viabilidad de las normas morales, a una ética de la responsabilidad consecuencial y pragmática. Pasamos de una regla de prudencia aleatoria y egoísta a un principio de prudenciabilidad comunitaria, crítica y racionalmente fundamentado.

La responsabilidad moral posee dos sentidos o dos momentos distintos. Actuar de forma éticamente responsable significa, en primer lugar, considerar, averiguar y asumir a priori las consecuencias de una norma ética. Esto se funda en que en el proceso argumentativo se reconocen a priori los intereses de todos los posibles afectados. ${ }^{2}$ En segundo lugar, actuar responsablemente significa asumir el carácter "ideal" del principio ético, y por tanto, su diferencia o contradicción con las estructuras,

${ }^{2}$ Cf. Karl-Otto Apel, "La ética del discurso como ética de la responsabilidad. Una transformación postmetafísica de la ética de Kant", Teoría de la verdad y ética del discurso, Ed. Paidós, Barcelona, 1991, p. 178. 
Mario Ramírez

procedimientos y normas de los consensos efectivos de la comunidad real. ${ }^{3}$ Comportarnos sólo en función de la norma ética -especialmente si se trata de un comportamiento social-cuando no podemos suponer que todos los demás miembros de la comunidad lo hagan, y cuando más bien podemos suponer que actúan sólo estratégicamente, puede ser un comportamiento moralmente irresponsable.

Para una ética discursiva, en tanto ética crítica, tan problemática y cuestionable es una acción estratégica "inmoral", o por lo menos axiológicamente neutral, como una moral pura pero dogmática e inviable prácticamente. La función del discurso, de la interrrelación humana racional, es la de mediar críticamente el deber moral postulado intuitiva y dogmáticamente en la esfera de la "eticidad sustancial" (las formas de la moralidad tradicional). El diálogo racional determina las condiciones y posibilidades efectivas del deber, es decir, lo fundamenta racionalmente. Por ejemplo, el "respeto a la dignidad de la persona" deja de ser un imperativo abstracto, fundado en una mera intuición y remitido a una suposición metafísica o teológica acerca de la "naturaleza humana", para

72 convertirse en un principio pragmáticamente redefinido como "reconocimiento de la capacidad comunicativa de cualquier ser humano". $\mathrm{El}$ deber para con el otro es explicado en términos de interacción social, de reciprocidad asumida, contextuada y desplegada colectivamente. El deber es comprendido, pensado, razonado. Esto no significa imposibilitarlo; significa mostrar cómo puede ser vital y comunitariamente necesario y posible. De ahí que una ética de la responsabilidad sólo pueda ser discursiva, pública y dialógica.

Que la responsabilidad es un a priori para una ética discursiva significa quela búsqueda de las condiciones de aplicación del principio de universalización determina el primer compromiso práctico del sujeto ético. La norma ética nos exige transformar las condiciones de la comunidad real, superando los obstáculos que nos impiden actuar bajo el principio de universalización de la comunidad ideal. Como lo explica Apel, "en la

${ }^{3}$ Cf. op. cit., p. 182.

${ }^{4}$ Cf. Adela Cortina, "La ética discursiva", en V. Camps (ed.), Historia de la ética, t. III, Ed. Crítica, Barcelona, 1989, p. 536 y 550. 


\section{LA NORMA ÉTICA UNIVERSAL}

medida en que ha aceptado ya siempre las condiciones ideales anticipadas de la fundamentación procedimental de normas como éticamente obligatorias para regular los conflictos en el mundo real, y teniendo en cuenta la diferencia que también ha de aceptar entre las condiciones ideales y las reales, el que argumenta ha aceptado también necesariamente la obligación moral de ayudar a superar la diferencia -a largo plazo, aproximativamente-mediante la transformación de las relaciones reales". ${ }^{5}$ Estamos obligados tanto a orientar éticamente nuestras acciones estratégicas como a posibilitar estratégicamente nuestras decisiones éticas. Tal es la situación completa de la condición humana: un movimiento circular y continuo de la "comunidad ideal" a la "comunidad real", de la racionalidad ética a la racionalidad estratégica (de la parte A de la ética a la parte B y de la B a la A). Tal es la paradoja de la moralidad humana: la inmoralidad puede producirse tanto por una acción estratégica irresponsable (la inmoralidad propia del materialista) como por una acción ética estratégicamente infundada (la inmoralidad propia del idealista). Por lo tanto, un concepto completo de la racionalidad ética consiste en "la complementación de la norma básica ética de la racionalidad discursiva a través de un principio de racionalidad estratégica, que a su vez se encuentra bajo un telos ético" ${ }^{6}$ En el propio Apel se puede reconocer un movimiento que va de una concepción que insiste en la oposición irreductible entre la racionalidad estratégica y la racionalidad comunicativa, a una concepción que busca la síntesis de ambas, en cuanto consistencia última y efectiva de la "racionalidad ética".

${ }^{5}$ Karl-Otto Apel, "¿Límites de la ética discursiva?", epilogo de Adela Cortina, Razón comunicativa y responsabilidad, ed. Sígueme, Salamanca, 1985, p. 254.

${ }^{6}$ Karl-Otto Apel, "¿Es posible distinguir la racionalidad ética de la racionalidad estratégico-teleológica?", en Estudios éticos, Ed. Alfa, Barcelona, 1986, p. 100. Cf. en esta misma obra el trabajo "iEs la ética de la comunidad ideal de comunicación una utopía?" 
Mario Ramírez

\section{La transformación de la comunidad real}

La ética discursiva se convierte así en un principio regulador crítico que nos permite interpretar, conducir y evaluar los procesos de la transformación social concreta, el dinamismo de la comunidad humana real. En este momento, ética y política se unen. La ética aparece como el principio rector de toda práctica social, como el elemento crítico que permite cuestionar toda concepción objetivista, positivista, autoritaria o metafísica de la realidad social y de los procesos de su transformación. Así, el criterio de una ética discursiva, en tanto ética social crítica, se puede enunciar de la siguiente manera: para poder aplicar la norma del consenso racional de la comunidad ideal debemos transformar las condiciones actuales de la comunidad real, pero esta transformación debemos realizarla bajo el telos normativo del principio ético. Es lo que Apel llama una "estrategia moral a largo plazo".

74 De lo anterior se sigue la necesidad de matizar el concepto de "transformación social". Podemos distinguir, en primer lugar, el nivel objetivo y el nivel subjetivo. El primero se refiere a las condiciones materiales, institucionales y funcionales de la vida colectiva. El segundo se refiere a aspectos de la vida humana que prevalecen como irreductibles a la esfera de la discursividad (la voluntad, la sensibilidad, los sentimientos, etc.) y que, según nuestro punto de vista, no son necesariamente contradictorios con la posibilidad de la discursividad, y deben, por tanto, ser considerados sin demérito por la "ética discursiva" (cosa que Apel no hace, al menos explícitamente).

Respecto al concepto de "transformación social objetiva" se deben distinguir dos momentos: el formal y el sustantivo.

1) La transformación formal: hacia la "comunidad ideal". Este momento se refiere a las transformaciones que debemos efectuar en la comunidad real a fin de aproximarnos lo más que podamos a la forma de la comunidad ideal. Se trata de superar aquellas situaciones actuales que niegan u obstaculizan la posibilidad de 


\section{LA NORMA ÉTICA UNIVERSAL}

una comunidad comunicativa libre y racional, y de establecer las condiciones institucionales, básicas y mínimas, para el logro de una participación social igualitaria. Los requisitos del consenso racional determinan teleológicamente aquellos aspectos de la sociedad actual que deben ser modificados y desarrollados.

2) La transformación sustantiva: desde la comunidad ideal. Este momento se refiere al conjunto de normas particulares, decididas comunitariamente y conforme a la norma ética básica, que van a regir las transformaciones específicas -económicas, sociales, políticas, culturales- de una sociedad concreta.

La relación entre los dos momentos de la transformación objetiva no es, sin embargo, de orden cronológico; la prioridad es lógica pero no empírica. Hay o debe haber simultaneidad entre ambos momentos. No podemos esperar hasta el establecimiento de la "comunidad ideal" para realizar transformaciones sociales. Pero tampoco podemos realizar estas transformaciones como si efectivamente fueran el producto de la "comunidad ideal" -pues implicaría recaer en el dogmatismo. Por lo tanto, las normas que rigen un proceso de transformación social deben estar sometidas a una confrontación crítica, a una revaluación y redefinición continua y sistemática. Implica también que la normatividad social debe permitir y respetar un margen de particularidad irreductible, así como definirse bajo una modalidad de "formalismo" nunca superable del todo.

Es por esto que la "comunidad humana ideal" es definida por Apel como siendo, en principio, "comunidad ideal de comunicación". Para el pensamiento crítico la realidad de la mejor comunidad humana es algo que se encuentra permanentemente en discusión y construcción. La "comunidad ideal" no es una entidad o una mera representación. Una comunidad ideal humana tendría que ser aquella que se mostraría a sí misma práctica y pragmáticamente, en el proceso efectivo de las interrelaciones concretas de los hombres. Sería una comunidad capaz de autorreflexionarse, autodiscutirse y autoconducirse (una comunidad "perfecta" que no pudiera discutirse a sí misma no sería tal). De ahí el 
Mario Ramírez

lugar central de la comunicación humana: ella no es sólo el punto de partida de la "comunidad ideal" sino también su punto de llegada, su prueba y su criterio.

\section{Las condiciones subjetivas de la transformación social}

Finalmente, deseo señalar que, aunque Apel no desarrolla una explicación acerca de las condiciones específicamente subjetivas de una ética discursiva (aspecto "motivacional" del comportamiento ético), no obstante, puede desprenderse tal explicación a partir de algunos señalamientos marginales de su reflexión. La "tranformación subjetiva" de la comunidad real debería darse, al igual que la estratégico-objetiva, de acuerdo con el ideal regulativo de la norma ética básica-pero tendrá que darse.

Nos referimos ante todo a la función insuperable de la voluntad en el proceso de la formación ética de la humanidad. En general, creemos que una filosofía de la "intersubjetividad", como la de Apel, no anula o tacha la "subjetividad" (como experiencia individual), simplemente la redefine en un contexto interactivo y comunicativo. Como señalaba MerleauPonty respecto a Heidegger, la subjetividad es "indeclinable", aunque se encuentre "mediada"; pues sólo ella nos asegura que la "comunidad ideal" es, críticamente, una "comunidad intersubjetiva", viva y abierta, y no una "estructura suprasubjetiva" (una estrutura anónima, abstracta y cerrada a la cual los sujetos estarían mistificadamente subordinados).

De esta manera, a la preocupación planteada por Apel respecto a que si puedo contar con que los demás actúan éticamente, se puede agregar una cuestión más: ¿puedo contar con que quieran actuar éticamente, es decir, qué tengan voluntad para el diálogo y la argumentación? Apel ha reconocido que la función de la voluntad es indeclinable aun para una ética discursiva. ${ }^{7}$ La elección por la razón puede ser explicada por la

7 "Aquí -cuando se trata de de ratificar voluntariamente el conocimiento de la validez- la ética discursiva tiene que reconocer -como ya hiciera Kant-como límite suyo (si queremos decirlo así) el 'misterio' irracional de la libre decisión de la voluntad (itambién para el mal!)". K.-O. Apel, "iLímites...?, op. cit., p. 234. 


\section{LA NORMA ÉTICA UNIVERSAL}

razón (no es un acto de fe), pero la voluntad para ratificar la elección racional no puede ser explicada o construida, a menos que se quiera negar el sentido último de la autonomía y la libertad moral. La norma ética universal -la posibilidad del consenso racional-se encuentra presupuesta en las estructuras pragmáticas de la comunicación y no depende, por tanto, de un acto voluntario (la norma se impone independientemente de nuestra voluntad desde el momento que ejercemos comunicativamente el lenguaje). No obstante, la norma es solamente una presuposición, una condición de posibilidad; el acto lingüístico no es de por sí un "hecho moral"; es imprescindible que el sujeto lingüístico asuma reflexiva, autónoma y voluntariamente las condiciones e implicaciones de su actuar y esté dispuesto a sostenerlas consecuentemente.

Ahora bien, ¿no cabe pensar, análogamente con la preparación estratégica de la racionalidad ética, una preparación comunitaria de la "subjetividad", una educación de la voluntad para el diálogo? ¿Y cómo debería ser? No podría consistir en una reafirmación de las normas de la "eticidad sustativa" y de las "decisiones" personales, puesto que es precismente aquello que está en cuestión para una ética discursiva. ${ }^{8}$ Tendríamos que remitirnos a la posibilidad de una interacción comunitaria "vital" aunque no sustantiva o particular; comunicativa, consensual incluso, aunque no discursiva ni argumentativa. Tal posibilidad, creemos, es la de la "comunidad estética". Queremos recordar que la capacidad humana para el diálogo, la comunicación y la humanización también es una cuestión de sensibilidad y no sólo de inteligencia o discurso.

La dimensión estética de la comunidad guarda una distancia equilibrada respecto a la acción estratégica y respecto a la acción ética, respecto a la comunidad particular y respecto a la comunidad ideal. La intuición estética, como intuición de una "idea infinita actual", y la formación comunitaria de la voluntad a través de las interacciones "expresivas"

${ }^{8}$ Apel ha confirmado esta tesis, aunque atenuada, en su reciente crítica al comunitarismo. Cf. la ponencia "La intención del 'comunitarismo' anglo-americano desde la perspectiva de la ética del discurso", leída en el Simposium Internacional "500 años: un pensamiento sin fronteras", Toluca, Octubre de 1992. 
Mario Ramírez

(artísticas y culturales en general) son las condiciones subjetivạs de una transformación de la comunidad actual hacia la comunidad ideal, pero son también una anticipación realizativa -experimental-del ideal comunitario para toda comunidad actual.

Si queremos mantener el proyecto de la ética discursiva, en tanto ética universal, racional e intersubjetiva, evitando eficazmente los peligros de formalismo, idealismo o intelectualismo, debemos reconocer por una parte-como ya lo ha hecho Apel- la necesidad de un proceso de acción estratégica (objetivo-social), en cuanto proceso que al mismo tiempo se encuentra orientado por el telos de la normatividad ética y es, no obstante, su condición de posibilidad práctica; y, por otra parte, la necesidad también de un proceso de acción estética -que no reconoce Apel-en cuanto condición subjetiva de la realización de la comunidad ética. La experiencia estética es la única forma de experiencia subjetiva e intersubjetiva, concreta y vital, que es no contradictoria con las exigencias de racionalidad y universalidad de la ética de Apel; es la fuerza y la sustancia vital sin la cual la éstetica discursiva permanecería en formalismo intelectualista o utopismo precavido, en moralidad "inaplicable".

Finalmente, nuestro planteamiento tiene por consecuencia inmediata apuntar, desde la perspectiva de la ética discursiva, a la revisión del concepto de "estética" en la tradición del pensamiento moderno. Recordemos que ya Kant señalaba la necesidad de tender un puente entre una teoría formal de la razón práctica (ética deontológica) y una ética material y teleológica, a través, precisamente, del análisis del "juicio estético" (la tercera Crítica). Recordemos también que antes de la emergencia y consolidación del universalismo teleológico hegeliano, los pensadores postkantianos, especialmente Schelling y Schiller, habían vislumbrado en la experiencia estética la posibilidad de una síntesis no acrítica ni dogmática entre el mundo de la Ley moral y el mundo de la Inclinación y la Pasión. 